\title{
Effective Internet Traffic Management by Reducing Congestion in TCP Cubic through Proactive Approach
}

\author{
Ahmed Mateen \\ University of Agriculture \\ Deptt of Computer Science, \\ Faisalabad, Punjab, Pakistan
}

\author{
Jawad Ahmad \\ University of Agriculture \\ Deptt of Computer Science, \\ Faisalabad, Punjab, Pakistan
}

\begin{abstract}
The future of modern Internet is adapting Cloud Computing, IoT and 3D technology over the Internet. So, Internet needs to exchange a huge amount of information in the form of packets of requests, responses and controlling data. An Internet connection always has finite throughput and can only support certain number of packets at a time. Transferring too many packets on Internet connection can produce heavy Internet Traffic which may cause bottleneck or network congestion. Meanwhile, the operations between the communication devices also effect and slow down. Effective congestion control is one of the most critical issues in the efficiency of Internet Traffic Management. To make it sustainable and efficient, a systematic approach to planning, testing and maintaining its behavioral mechanism is desirable to avoid network congestion and keep the data flowing smoothly. In this area of traffic management, the research will be conducted on different TCP variants and proposed a model of congestion avoidance. The new Congestion Avoidance algorithmic model will enhance the performance and utilization of the network, by making TCP more flexible and efficient protocol with a new proactive approach. The results of analysis will help the industrial developers and researchers to implement the proposed solution in Linux Kernel for more stable version of TCP
\end{abstract}

\section{Keywords}

TCP, CUBIC, Congestion Avoidance, Congestion Control, TCP Variants, Internet Traffic Management, Network Congestion,

\section{INTRODUCTION}

The modern Internet has revolutionized the computer and electric communications world like nothing before [1]. Now the Internet is a world-wide broadcasting medium and a method for information distribution, collaboration and communication between human being and their computers without concern for geographic location [2]. Today the day-to-day activities of computing world mostly rely on internet. Almost every big organization in the world is using Internet services for its operation and communication.

The Internet is a high extensive network of networks, such as the network infrastructure. It connects millions of computers around the world together and forming a huge network in which a computer can communicate with another computer as long as they want to communicate, and connected to the network Internet. Nobody owns the Internet, and not one person or organization controls the Internet. Internet is a more than the same concept of a material object, and is based on the physical infrastructure that connects networks other networks.[3]

The scientist predict that one day the modern Internet will become 'like electricity', less visible, however more deeply surrounded the people's lives. The future of modern Internet is adapting Cloud Computing, 3D Technology, Internet of Things, Global Connectivity, Grid Computing, Wireless Media and HD Multimedia Broadcasting over the Internet. To achieve the needs of these technologies, there are lots of communication devices accessing resources and getting request to carry out their work on Internet. So, Internet needs to exchange heavy information in the form of requests, responses and controlling data.

As discussed earlier the Internet is a worldwide network which connects billions of electronic devices. The Electronic devices may be Servers, Computers, Laptop, Mobiles, Tablet, Fax, Printers, IPTV, and other media which send or receive the data through a network. At this stage more than 190 countries are interconnected for the exchange of data, information, and ideas. According to survey report of Internet Live Stats Department, there was an approximately 3,037,608,300 Internet users globally till the December 30, 2014. This figure of Internet users shows that it is approximately $40 \%$ of population of the world. The major part of the Internet users is in China, followed by the United States and after Europe.

Most of the old media communication, including telephony and television, reformed or processed online because the birth of new technologies and services like Voice over Internet Protocol Television (IPTV) and Internet Protocol (VoIP) However, newspapers, books and many other printed publications and adaptation of technology on the site, transformed into blogs and Web feed, Industrial entertainment, including music, movies and games are also the main causes of the strongest growth in Internet.

\subsection{BACKGROUND OF PROBLEM}

Before discussing the issues of Internet traffic, it is necessary to clear the basic concept of Internet traffic [4]. It can be defined in many ways like Internet traffic is kind a load on communication devices or networked system. Mostly the Internet traffic is exists on the internet in shape of accessing the World Wide Web (www) traffic that flowed between web servers and web clients and File Sharing Traffic. So the Internet Traffic is a density of data which travels on the Internet Connection. Internet exchanges numerous information in the form of a huge number of:- 
- Packets of requests

- $\quad$ Packets of responses

- Packets of instructions

Intel conducted a survey and reported that "what is happening in an Internet minute". More than 23000 hours of video watched at Netflix, 0.138 million hours of video watched at YouTube, 4.1 Million Searches done at Google, 3.3 Million content shared [5]. These two multimedia websites is generating more than half of total internet traffic. Today we can say that the number of connected electronical devices is equals the world's population. In 2020, the number of connected devices is expected to be triple of the world's population [6].

One of most major issue is, the growth of Internet Traffic is increasing tremendously because:-

The demand of new evolving technologies increasing[1].

- The number of connected devices increasing.

- The number of users also increasing.

- More \& More uses of online resources.

- For Global distribution of knowledge \& information.

- Computing power is touching everybody due to wireless technology \& mobile devices.

\subsection{QUESTION ARISES}

Here some questions arises that:-

- Can the existing networks handle this explosion of network traffic?

- Can it accommodate this growth while maintaining security?

- Can it maintain consumer expectations for instant access to online resources?

- Can the existing networking infrastructure support the Cloud Computing?

- Can new software technologies and applications run smoothly on this network?

- Can the wireless network upgrade on this existing network?

- Why its management and enhancement is so necessary?

\subsection{PROBLEMS STATEMENT}

The future of modern Internet is adapting Cloud Computing, IoT and 3D technology over the Internet. So, Internet needs to exchange a huge amount of information in the form of packets of requests, responses and controlling data.

The basic major issue is that the growth of Internet Traffic is increasing tremendously and on the other hand, the Internet connection always has finite throughput for data flow and can only support certain number of packets at a time. It is difficult to transferring too many packets at the same time on Internet connection because sending too many packets at a time can produce heavy Internet Traffic which may cause bottleneck or Network Congestion. Meanwhile, the operations between the communication devices also effect and slow down. This also ultimately results in congestion of Internet. It may also deteriorate network service quality in the shape of:
- Queuing delay

- Frame or data packet loss

- Blocking of new connections

- $\quad$ Reducing the rate of good put

\subsection{SIGNIFICANCE OF INTERNET TRAFFIC MANAGEMENT}

The Effective Internet Traffic Management is the solution to eliminate these problems and keep the data flow smoothly. It is also like a road traffic control. Traffic Engineers / Network Admins control Internet Traffic and define different schemes to implement, how to avoid or reduce network congestion? The number of users and connected devices increasing and Internet Traffic Management shows its significance when talked about the demand of new evolving technologies like Cloud Computing, 3D Video Streaming, Internet of Things, Global Connectivity, Grid Computing, High Speed Wireless Media, and HD Multimedia Broadcasting [15]. These technologies require high computing powers which relatively demands high bandwidth internet speed for resource sharing.

Internet traffic management is a research area within the Communication Networks and Systems Engineering. Internet traffic congestion can be a nightmare for Network Engineers and Data Centre Managers but not, if they deals it with proper Traffic Management System. Effective congestion control is one of the most critical issues in the efficiency of Internet Traffic Management. To make it sustainable and efficient, a systematic approach to planning, testing and maintaining its behavioral mechanism is desirable to avoid network congestion and keep the data flowing smoothly [7][9].

\subsection{OBJECTIVES}

To make Internet Traffic Management sustainable and efficient, a systematic approach to planning, testing and maintaining its behavioral mechanism is desirable to avoid network congestion and keep the data flowing smoothly. Effective congestion control is one of the most critical issues in the efficiency of Internet Traffic Management which needs to be resolved.

\subsection{POSSIBLE SOLUTIONS}

Bandwidth management which is a technique of Internet Traffic Management is a kind of procedure of quantifying, monitoring and governing the communications system like traffic in shape of frames or packets on a network link [8]. Bandwidth management is used to avoid to overfilling the network link, which may cause the network congestion or poor performance of the network.

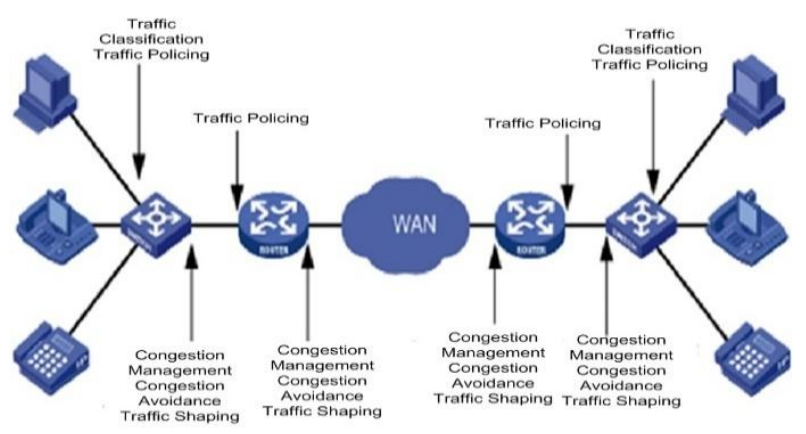

Figure 1: The Internet Traffic Management 
Internet traffic can be managed by Bandwidth management mechanisms which may further be used to check the performance of network and controlling the network congestion [14]. Those mechanisms include the following schemes:-

- Traffic shaping (rate limiting)

- Scheduling algorithms

- Congestion Control algorithms

- Congestion avoidance

Congestion can occur when data arrives on the big pipe (fast LAN) and is a small tube (WAN slower) [12]. Overload can also occur when multiple input streams arrive at a router whose output capacity is less than the sum of inputs. To avoid overloading a lost packet inspection method is used which is called Congestion Avoidance.

\section{RESEARCH METHODOLOGY}

In this area of traffic management, the research has been conducted on different TCP variants to enhance the performance \& utilization of the network, by making TCP more flexible, efficient \& stable protocol. In this thesis, the Internet Traffic Congestion has been dealt with Proactive approach by

- Planning, Organizing, and defining the Problem.

- Measuring the Internet Traffic

- Analysis of different Linux TCP variants

- Simulation of TCP Variants specially TCP Cubic

- Changing its behavioral mechanism

- $\quad$ By reducing the Packet Size

- Controlling congestion window size

- $\quad$ Fast Recovery \& Retransmit

- $\quad$ Testing the proposed solution,

- $\quad$ Final results and findings.

- Simulation on Dumbbell Topology

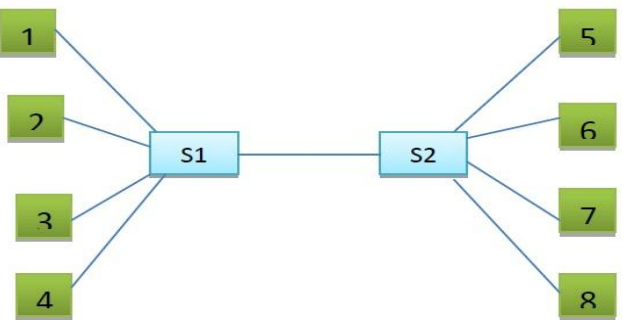

Figure 2, Simple Dumbbell Topology

The cycle is determined by the NS2 simulator. The throughput is measured in kbps to receive weights for each version of High Speed TCP. In our modeling packet size set to 1000 in all cases. The traffic flow in the dumbbell network will simulated and analyzed using different TCP variants such as: TCP Tahoe, TCP Reno, TCP New Reno, SACK, HSTCP, STCP, CUBIC to assess the presentation obtainable through the divergent of variants of the TCP realized in Linux version of 2.6. The complete record of the TCP Linux variants, representing the type of network and the relevant variant of TCP which has been chosen for this network towards execute the under study experiment here are worn couple of tackle, equally liberally.

In the part of Traffic control and management, normally two approaches uses to the handling of Congestion

First is

- Congestion Control which is (Reactive approach) Play after the network is overloaded

Second is

- Congestion Avoidance which is (Proactive approach) Play before the network becomes overloaded

In reactive approach It always do after the network is congested or overloaded [10]. Whereas in proactive approach It do before occurring the network congestion. There are two types of network congestion handling methods, Hardware Based and Software Based.

\section{RESULTS}

As a result of experiments, it was shown that TCP CUBIC has advantageous properties compared to TCP Reno and TCP latest options for high-speed networks and long overdue. The cubic TCP implementation can be found on the Internet. From the experimental results in this work it is shown that the throughput of TCP CUBIC strongly depends on the measurement result of the available bandwidth of the network online by measurement. Thus, it is intended to improve the built-in filter mechanism measurements of the network, taking into account the effect of the core resolution timer. In addition, the measurement algorithm is based on the bandwidth and the packet arrival frequency is the significant difficulty in high-speed networks.

Table 1, Throughput of Different TCP Variants

\begin{tabular}{|c|c|}
\hline TCP Variants & Throughput (Kbits/s) \\
\hline Reno & 305 \\
\hline New-Reno & 410 \\
\hline HSTCP & 413 \\
\hline STCP & 393 \\
\hline SACK & 405 \\
\hline BIC & 390 \\
\hline FAST & 350 \\
\hline CUBIC & 440 \\
\hline
\end{tabular}

This evaluation of the representation of the high-speed versions of TCP throughput positions using the network weights. This test, the presentation TCP depends mainly on the load on the network [13]. From this simulation results, TCP CUBIC works best for congestion management in Linux based operating systems. In this study, it has not been considered the other limiting performance, such as speed, convergence and TCP RTT fairness of the judiciary. Moreover, we have to see eye the presentation of TCP options at high speed with strong restrictions to improve the performance of TCP-weights 


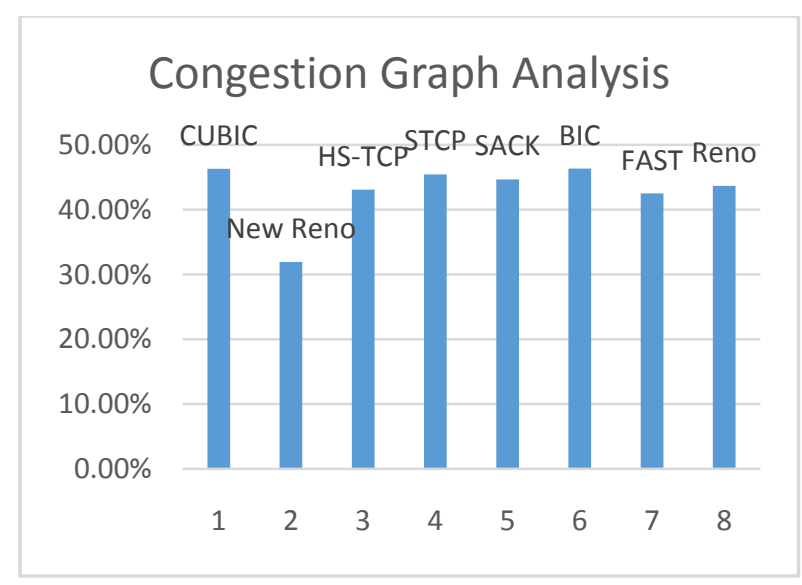

Figure 3, Throughput and Congestion Avoidance Graph

By analyzing the above mentioned results the following model is proposed for the new Congestion Avoidance algorithm.

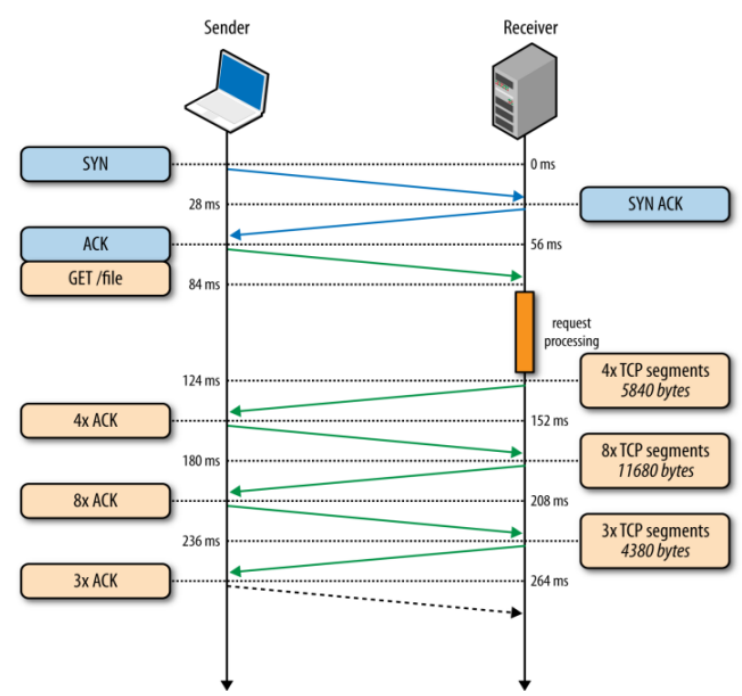

Figure 4, Proposed Congestion Avoidance Alogorithm

The behavior and performance of TCP CUBIC were multiple clusters control regime as TCP Reno with BIC, scalability, high speed, and quickly, so compared CUBIC allow users to use the settings window. CUBIC manages and improves bandwidth allocation, equity, because the show will be taken inflows. Practice of NS-2 performance and compared to other TCP algorithms ie. TCP Reno, HSTCP, STCP and the TCP connection.

Deployment of the model was tested with several known properties of CUBIC as slow convergence and RTT fairness in dumbbell topology [11]. More importantly, the stability, of this new model of Congestion Avoidance provides support in concave areas although widely used in CUBIC. Given the growth process window as a random process, the model proposed, derived from a Markov chain. Although the size of the window, the duration and the number of variable before deleting stored packets._In detail this mechanism elaborated as under:-

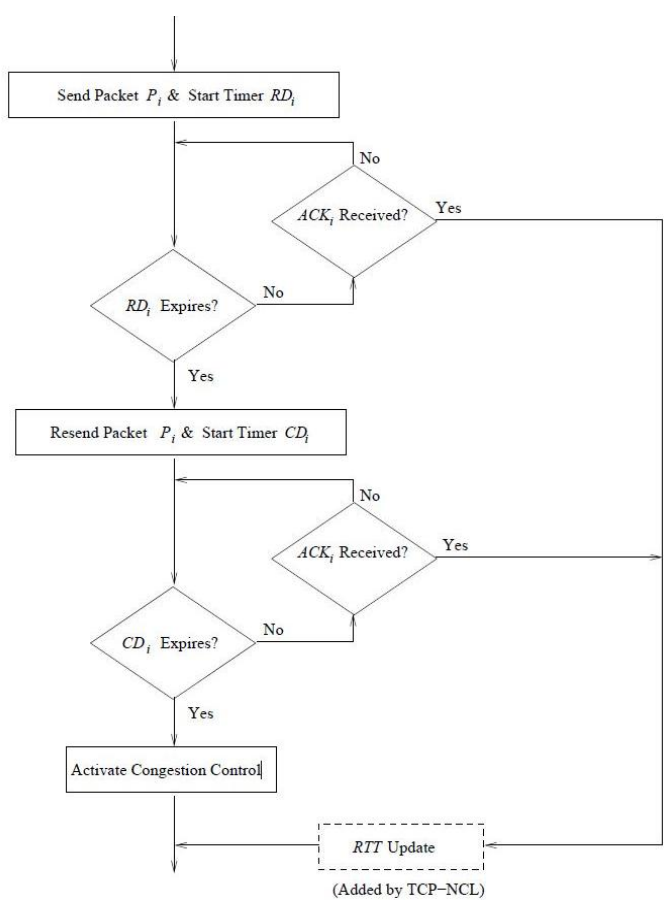

Figure 5, Flow Chart of new Congestion Avoidance Algorithm

After implementation, the result is estimated by simulation experiments with above mentioned new congestion control mechanism, network-based on-line measurement in high speed networks. Many simulation results comes but only showed here the effectiveness of broadband networks mechanism. It is confirm that the new Congestion avoidance mechanism is better than other existing TCP congestion control mechanisms in terms of bandwidth and small enough tie the queue length of a narrow, while almost no packet loss. The results of the analysis of the simulation results showed here in the shape of Throughput Analysis graph.

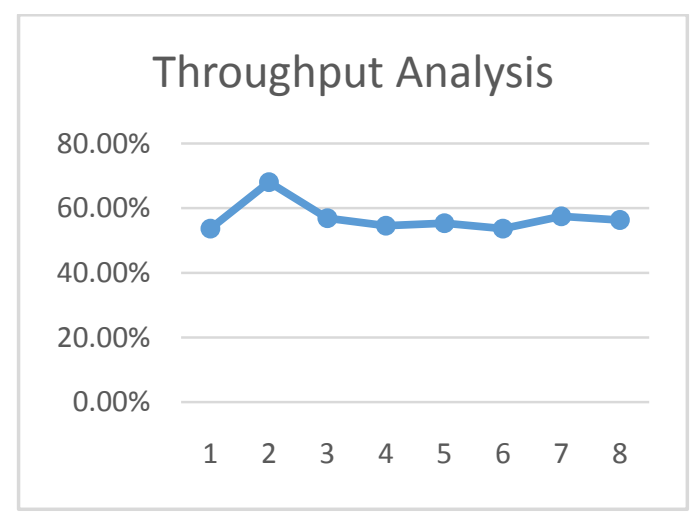

Figure 6, Throughput and Congestion Analysis

The performance after implementation of this model in TCP CUBIC will appreciate over public Internet environment in Linux bases Operating Systems. CUBIC TCP uses an approach based on bandwidth: it adjusts the size of the congestion window and update its RTT based on the information of the physical capacity and available bandwidth.

\section{CONCLUSION / FUTURE WORK}

The research work can be divided in to two parts. In the first part, a thorough study and theoretical baseline has 
been developed on Internet Traffic Management, started with a preliminary investigation of the relationship between Internet Traffic Management and Congestion. In second part TCP congestion control protocol and its variants has been discussed by doing some experimental approach on NS-2. On the bases of their results and analysis as mentioned above, a new Congestion Avoidance protocol has been proposed for the current and future Internet Traffic Management for the reliability and stability of TCP in Linux Operating System. TCP CUBIC is able to adopt this stable model of congestion avoidance under nontrivial end-to-end communication delays. In the area of traffic management, studies have been conducted on various TCP options to improve performance and network utilization by TCP CUBIC flexibly and efficiently with a proactive approach. The analysis results help industrial designers and researchers to implement the proposed solution for Linux Kernel for a stable version of TCP CUBIC

\section{REFERENCES}

[1] Abed, S. A., 2013. Comparative Analysis of TCPProtocol Operation Algorithms in Self-Similar Traffic. Journal of Communications and Network, 5(2): 1-9.

[2] Brett, L., 2014. A TCP CUBIC implementation in ns3. Proceeding, WNS3 14 Proceedings of the 2014 Workshop on ns-3, 1(3):5-15.

[3] Darrell, M. W., 2014. The Evolution of Video Streaming and Digital Content Delivery. Journal of Technology Innovation at Brookings, 4(13):80-86.

[4] Federico, B., 2010. Internet in the development of future road-traffic control systems. Internet Research Journal, 20(2): 154-168.

[5] Feknous, M., 2014. Internet traffic analysis: A case study from two major European operators. IEEE Symposium on Computers and Communication (ISCC), 13(2): 124-130.

[6] Intel, C., 2014. What is happening in Internet Minute. Journal of Intel Corporation, plateform for enterprises, 1(13): 1-6.
[7] Jingyuan, W., 2013. CUBIC-FIT: A High Performance and TCP CUBIC Friendly Congestion Control Algorithm. IEEE Communications Letters, 17(8): 21-29.

[8] Mahida, 2013. A Comparative Analysis of Queue Management Techniques using NS-2 Simulator. International Journal of Computer Applications 65(6):1-8.

[9] Marco, C., 2011. Research challenges towards the Future Internet. Computer Communications, 34(18): 2115-2134

[10] Martin, H., 2013. Advances in TCP Congestion Prevention. IEEE 11th International Symposium on Applied Machine Intelligence and Informatics, 8(5): 556-561

[11] Peng, Y., 2014. TCP Congestion Avoidance Algorithm Identification, IEEE/Acm Transactions On Networking, 22(4): 1311-1325.

[12] Sahuquillo, 2013. An Effective and Feasible CongestionManagement Technique for HighPerformanceMINs with Tag-Based Distributed Routing, IEEE Transactions on Parallel and Distributed Systems, 13(2): 124-130

[13] Tizghadam, A., 2010. Autonomic traffic engineering for network robustness. IEEE Journal on Selected Areas in Communications, 28(1):39-50.

[14] Vandana, K., 2014. Congestion control for highspeed wired network: A systematic literature review. Journal of Network and Computer Applications, 45(1):62-78.

[15] Yuanni, W., 2012. Fairness issues with TCP congestion window. Information Science and Digital Content Technology (ICIDT), 8th International Conference, 12(7):149-155. 